\title{
A Particular non-Atomistic Orthomodular Poset
}

\author{
R. J. GREECHIE \\ Department of Mathematics, Kansas State University, Manhattan
}

Received April 28, 1969

\begin{abstract}
Every atomic orthomodular lattice is atomistic. We show that the corresponding statement for orthomodular posets fails. The result is of interest in the study of the Algebraic Structure of Quantum Mechanics. see [5].
\end{abstract}

\section{Section 1. Introduction}

A partially ordered set (henceforth called a poset) $P$ with a smallest element 0 is atomic if every non-zero element of $P$ dominates an atom (a minimal non-zero element) of $P[7]$; it is atomistic if (it is atomic and) every element of $P$ is the supremum of the atoms which it dominates [6]. Not all authors agree with this terminology [2]; even worse, some do not explicitly define the term utilized.

Puzzled by [5], page 267, line 36 the present author posed the following question: Is every atomic orthomodular poset $P$ atomistic? The answer is negative (although if $P$ were a lattice the answer would be affirmative). Consider two copies of the power set of the integers and identify corresponding finite and co-finite subsets; with the induced order and orthocomplementation this is an atomic orthomodular poset (which is not a lattice). Any element of this poset which corresponds to an infinite and co-infinite subset of the integers has the property that it is not the supremum of the atoms it dominates; hence the poset is not atomistic.

However the Ref. [5] suggests that the additional assumption that every element $x$ of $P$ be finite (every chain from 0 to $x$ is finite) or cofinite ( $x^{\prime}$ is finite) may lead to an affirmative answer to the above question. It is the purpose of this paper to prove that this additional assumption does not remove the pathology; in fact, we present an atomic orthomodular poset which satisfies axioms A.1-A.8 of [5] but is not atomistic (in our sense).

\section{Section 2. The Poset}

Undefined terms appear in [2] or [3].

Let $Z$ denote the integers. Let $Z_{1}$ and $Z_{2}$ be disjoint copies of $Z$; for $i=1,2$ let $\phi_{i}: Z_{i} \rightarrow Z$ be the natural bijections; let $X=Z_{1} \cup Z_{2}$ $\cup\{-\infty, \infty\}$ where $-\infty$ and $\infty$ are any distinct elements not in $Z_{1} \cup Z_{2}$. 
Call a subset $M$ of $X$ admissible in case $M$ is a finite or co-finite subset of exactly one of either $Z_{1} \cup\{-\infty\}$ or $Z_{2} \cup\{\infty\}$. Let $\mathfrak{A}$ denote the collection of admissible subsets of $X$. Define an equivalence relation $\equiv$ on $\mathfrak{A}$ as follows:

For $M, N \in \mathfrak{A}, M \equiv N$ if and only if at least one of the following obtains:

(i) $M, N \in\left\{Z_{1} \cup\{-\infty\}, Z_{2} \cup\{\infty\}\right\}$;

(ii) $M$ and $N$ are finite, $M \subset Z_{1}, N \subset Z_{2}$, and $M \phi_{1}=N \phi_{2}$;

(iii) $M$ and $N$ are finite, $M \subset Z_{2}, N \subset Z_{1}$, and $M \phi_{2}=N \phi_{1}$;

(iv) $M$ and $N$ are infinite, $-\infty \in M, \infty \in N, M \backslash\{-\infty\} \subset Z_{1}, N \backslash\{\infty\} \subset Z_{2}$, and $(M \backslash\{-\infty\}) \phi_{1}=(N \backslash\{\infty\}) \phi_{2}$;

(v) $M$ and $N$ are infinite, $\infty \in M,-\infty \in N, M \backslash\{\infty\} \subset Z_{2}, N \backslash\{-\infty\} \subset Z_{1}$, and $(M \backslash\{\infty\}) \phi_{2}=(N \backslash\{-\infty\}) \phi_{1}$;

(vi) $M=N$.

Let $P$ denote $\mathfrak{A} / \equiv$; for $M \in \mathfrak{A}$, denote the corresponding element of $P$ by $[M]$. Partially order $P$ by defining for $[M],[N] \in P,[M] \leqq[N]$ in case there exists $[Q] \in P, Q_{i}, Q_{j} \in[Q], M_{i} \in[M], N_{j} \in[N]$ such that $M_{i} \subset Q_{i}$ and $Q_{j} \subset N_{j}$; write $[Q]:[M] \leqq[N]$. Noting that $[\phi] \leqq[M]$ $\leqq\left[Z_{1} \cup\{-\infty\}\right]$ for all $[M] \in P$, we define $0=[\phi]$ and $1=\left[Z_{1} \cup\{-\infty\}\right]$. Finally, we define an orthocomplementation ${ }^{\prime}: P \rightarrow P$ as follows: for $[M] \in P$,

$$
[M]^{\prime}=\left\{\begin{array}{lll}
{\left[\left(Z_{1} \cup\{-\infty\}\right) \backslash M\right]} & \text { if } & M \subset Z_{1} \cup\{-\infty\}, \\
{\left[\left(Z_{2} \cup\{\infty\}\right) \backslash M\right]} & \text { if } & M \subset Z_{2} \cup\{\infty\} .
\end{array}\right.
$$

In the notation of [4] the poset $P$ may be written

$$
P=\left(F C\left(Z_{1} \cup\{-\infty\}\right), F C\left(Z_{2} \cup\{\infty\}\right) ; S_{1}, S_{2}\right),
$$

where $F C(X)$ denotes the Boolean algebra of all finite or co-finite subsets of $X, S_{1}$ is the section of $F C\left(Z_{1} \cup\{-\infty\}\right)$ consisting of the ideal of all finite subsets of $Z_{1}$ together with the complements of such sets in $Z_{1} \cup\{-\infty\}$, and $S_{2}$ is the corresponding section of $F C\left(Z_{2} \cup\{\infty\}\right)$. Hence, by the theorem in Section I.(2) of [4], $P$ is an orthomodular poset. Some of its properties are listed in the following theorem.

Theorem. There exists an orthomodular poset $P$ such that

(1) $P$ admits a full set of states,

(2) $x \in P, x \neq 0$ implies $x$ is finite (every maximal chain from 0 to $x$ is finite) or $x^{\prime}$ is finite,

(3) $x \in P, x \neq 0$ implies $x$ dominates an atom of $P$,

(4) every Boolean sub-algebra of $P$ is countable,

(5) if $\{x, y, z\}$ is a pairwise compatible subset of $P$, then $x$ is compatible with $y \vee z$, and

(6) there exists a coatom $x \in P$ such that $\sup \{a$ : a atom, $a \leqq x\}$ does not exist. 


\title{
Bibliography
}

1. Birkhoff, G.: Lattice theory, Colloquium Publ., A. M. S., vol. XXV, 1967.

2. Greechie, R. J.: On the structure of orthomodular lattices satisfying the chain condition, J. Comb. Theory, 4, 210-218 (1968).

3. - Orthomodular lattices admitting no states, to appear in the J. Comb. Theory.

4. - On generating pathological orthomodular structures, to appear, The Symposium on Orthomodular Lattices, Univ. of Mass. Press (1970).

5. Gunson, J.: On the algebraic structure of quantum mechanics. Commun. Math. Phys. 6, 262-285 (1967).

6. Maeda, S.: On atomistic lattices with the covering property. J. Sci. Hiroshima Univ. Ser. A-1, 31, 105-121 (1967).

7. Szasz, G.: Introduction to lattice theory. 3rd ed. New York: Academic Press 1963.

\author{
R. J. Greechie \\ Department of Mathematics \\ Kansas State University \\ Manhatten, Kansas 66502, USA
}

